\title{
Systematic Design of Planar Lenses Using Artificial Dielectrics
}

\author{
Yan Zhang ${ }^{1,2}$, Raj. Mittra ${ }^{2}$, Wei Hong ${ }^{1}$ \\ ${ }^{1}$ State Key Laboratory of Millimeter Waves, School of Information Science and \\ Engineering, Southeast University, Nanjing, 210096, China \\ ${ }^{2}$ Electromagnetic Communication Laboratory, 319 EE East, Pennsylvania State \\ University, University Park, PA, 16802 \\ Email:yanzhang@emfield.org,Mittra@engr.psu.edu
}

\begin{abstract}
In this work we present a systematic approach for designing planar lenses using artificial dielectrics to realize the desired phase delays. The artificial dielectric is synthesized by using multilayer frequency selective surfaces (FSSs) with Jerusalem Cross (JC) elements, because they are found to be good candidates that provide stable response over a wide range of incident angles and for both polarizations.
\end{abstract}

\section{Introduction}

Although dielectric lens antennas provide desirable alternatives to reflectors in many applications, their use has been relatively limited at microwave frequencies because they are relatively heavy in weight and can also be difficult to fabricate. It is not surprising, therefore, that there is considerable current interest in designing planar lenses for microwave applications using metamaterials, or artificial dielectrics. Although a number of papers have appeared in the literature on this subject, no systematic design procedure for planar lenses has been presented to-date. The purpose of this paper is to show how we can use FSSs consisting of artificial dielectrics to realize controllable phase delays for lens function and to subsequently use them to synthesize a planar lens.

After considering a number of alternatives, we select the Jerusalem cross (JC) element for the FSS, because it exhibits frequency stable characteristics for a wide range of incident angles (up to $70^{\circ}$ ), and for both TE and TM polarization [1-2]. Although the magnitude of the frequency response characteristic of JC/FSS has been studied extensively in the past, the same cannot be said about their phase response behavior, which plays an important role when it is used to realize an artificial dielectric. This prompts us to begin by exploring the phase behavior of the transmission coefficient of the JC/FSS, with a view to assessing its suitability for planar lens design. We can control the above phase response by adjusting the geometrical parameters of the JC element. We then go on to show how we could utilize the knowledge of the phase response to systematically design a planar lens.

\section{Response characteristics of FSSs with Jerusalem cross elements}

Fig.1 shows the geometry of the JC element, which is comprised of a crossshaped conductor printed on a dielectric substrate $\left(\varepsilon_{\mathrm{r}}=2.2, \tan \sigma=0.0009\right)$, whose thickness is $0.5 \mathrm{~mm}$. Fig.2 depicts the model simulated by using the parallelized 
version of the FDTD software GEMS [4], in which the periodic boundary condition is utilized to model the FSS. As displayed in Fig.3, we can achieve a wide passband, centered around $30 \mathrm{GHz}$, when the element size $P$ is chosen to be $3.9 \mathrm{~mm}$. We observe the E-field is concentrated mainly around the top and bottom arms of the conductor at the first resonance, which occurs at $17 \mathrm{GHz}$, when the $\mathrm{JC}$ is excited by a TE wave with the E-field polarized along the $y$-axis. In contrast to this, at $30 \mathrm{GHz}$ the E-field is largely confined inside the substrate, a behavior that is consistent with the performance of the FSS in the passband. To systematically study the phase correcting function of the JC, we introduce a function called $P D$, which is defined as the incremental phase shift contributed by the JC in a wave that traverses through the FSS, and is expressed as:

$$
P D=\operatorname{Phase}\left(S_{21}{ }^{\text {vacuum }}\right)-\operatorname{Phase}\left(S_{21}{ }^{J C}\right)
$$

We observe from Fig. 3 that the $P D$ is nearly equal to $0^{\circ}$ around $30 \mathrm{GHz}$ and it changes approximate linearly in the neighborhood of this frequency.

Next, we study how the two geometrical parameters of the JC, namely $L 1$ and $L 2$ affect the $P D$, so we can utilize this knowledge to subsequently design the planar lens. Fig.4 illustrates the variation of the $P D$ with $L 1$ and $L 2$, and shows that we can achieve a phase increment ranging from $-10^{\circ}$ to $45^{\circ}$ by varying these parameters. To realize a wider range of phase variation, we can simply stack up multiple layers of this FSS, as may be seen from Fig.5(a), which presents the results for a two-layer case, with the layers separated by the distance $d z$. As expected, the mutual coupling between the two layers gets weaker with increasing $d z$, as evidenced by the fact that the two dips in $\left|S_{11}\right|$ move nearer to each other around $30 \mathrm{GHz}$. The two-layer case exhibits good transmission performance at this frequency, as it has both low loss and low reflection. By varying the geometrical parameter of the two layers, separated by $d z=2 \mathrm{~mm}$, we can obtain a phase increment $P D$ ranging from $-30^{\circ}$ to $120^{\circ}$, as may be seen from Fig.5(b). This leads us to observe that by using multi-layer FSS screens, separated by appropriate spacings, we can obtain the desired value of the $P D$ for designing a planar lens with good transmission performance.

\section{Zone-plate planar lens design procedure}

Next, we proceed to develop a systematic procedure for the problem at hand, namely designing the planar lens using the JC/FSS for the artificial dielectric. Fig.6(a) depicts a one-dimensional planar lens model, which is adequate for our purposes, in view of the symmetry of the lens geometry. The diameter and focal length of the lens are denoted by $D$ and $F$, and the phase shift caused by path difference can be approximately calculated from [3]:

$$
\phi(\theta)=\frac{2 \pi F}{\lambda_{0}} \frac{1-\cos \theta}{\cos \theta}
$$

where $\theta$ is the incident angle and $r$ is the distance from the incident point to the center of the lens. For this example, $F$ is chosen to be $6 \lambda$ ( $\lambda$ is the wavelength in free space) and $F / D=0.5$; hence, the incident angle $\theta$ ranges from $-45^{\circ}$ to $45^{\circ}$. Fig.6(a) plots the required phase as a function of $\theta$, and we note that we need a maximum value of the phase shift to be on the order of $900^{\circ}$, which is difficult 
to achieve by using the artificial dielectric we are considering in this work. This prompts us to consider a zone-plate structure so as to ensure that the maximum phase shift in any of the three zones is less than or equal to $360^{\circ}$, as shown in Fig.6(b) (the blue line). We find that for $30 \mathrm{GHz}$, and for JC element whose cell size is $3.9 \mathrm{~mm}$, altogether 31 elements are needed along the diameter $D$, which is $120 \mathrm{~mm}$ in size. The marked points on the green line in Fig.6(b) provide the sample values of the phase shift corresponding to the desired phase shift distribution of each JC element in the zone-plate lens. We can then determine the geometrical parameters of each element to design the final lens structure.

\section{Conclusion}

We have shown in this paper how we can systematically design a zone-plate planar lens for microwave frequencies using an artificial dielectric which we have synthesized by using Jerusalem cross FSSs. The designed lens has the desired characteristics of low-loss, low reflection and is lightweight as well.

\section{References}

[1] S. E. Melais and T. M. Weller, 'A multilayer Jerusalem cross frequency selective surface.' Wireless and Microw. Tech. Confer., pp. 1-5, Apr. 2009.

[2] C. R. Simovski, S. A. Tretyakov, and P. de Maagt, 'Artificial high impedancesurfaces: Analytical theory for oblique incidence.' in Proc. Antennas Propag. Soc. Int. Symp. vol. 4, pp. 434-437, 2003.

[3] N. Gagnon, A. Petosa, D. A. Mcnamara, 'Comparison between conventional lenses and an electrically thin lens made using a phase shifting surface at Ka band.' Antennas and Propaga. Conf. Loughborough, 2009, pp. 117-120, 2009.

[4] GEMS, 3-D High Performance parallel EM simulation software, www.2comu.com.

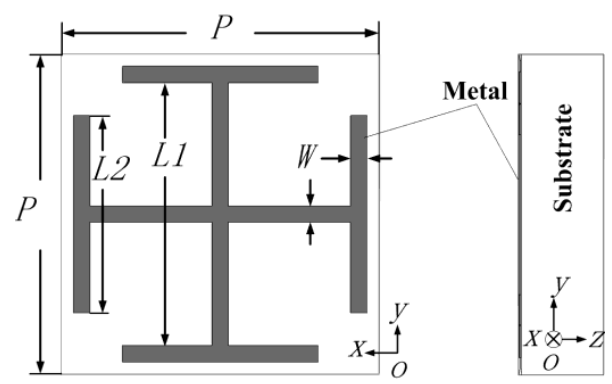

Fig.1. The geometry of the JC

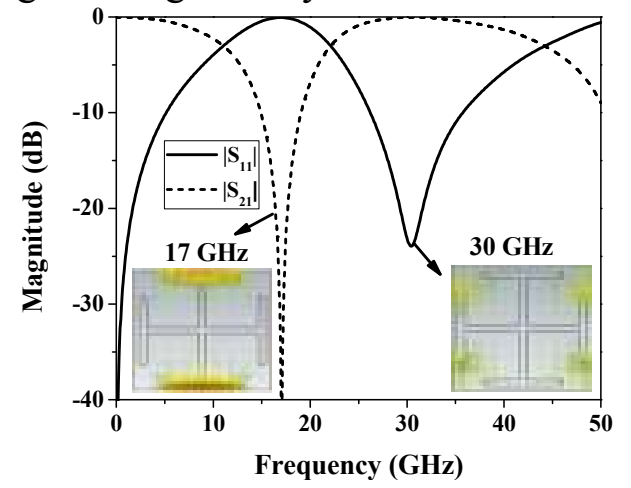

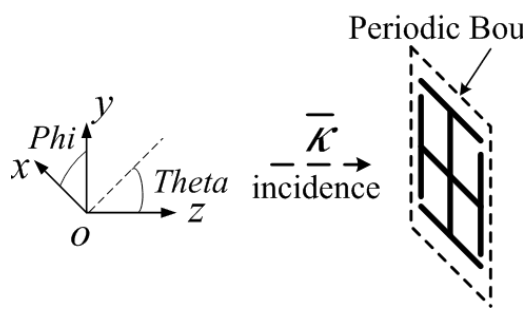

Fig.2. The simulation model

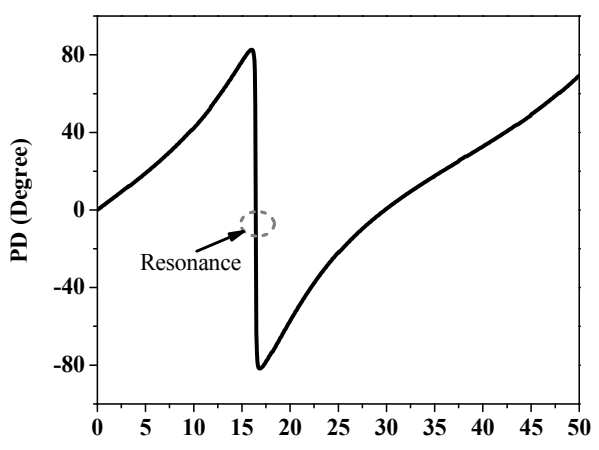

Fig.3. The transmission performance when $P=3.9 \mathrm{~mm}$ 

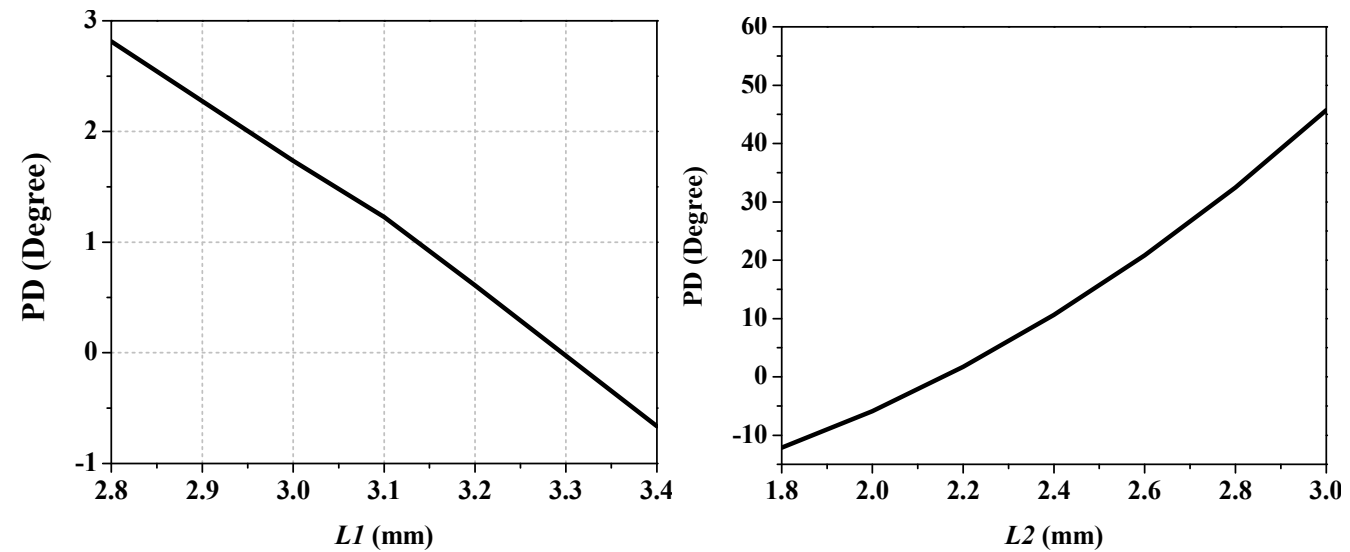

Fig.4. The JC's phase correcting property against $L 1$ and $L 2$

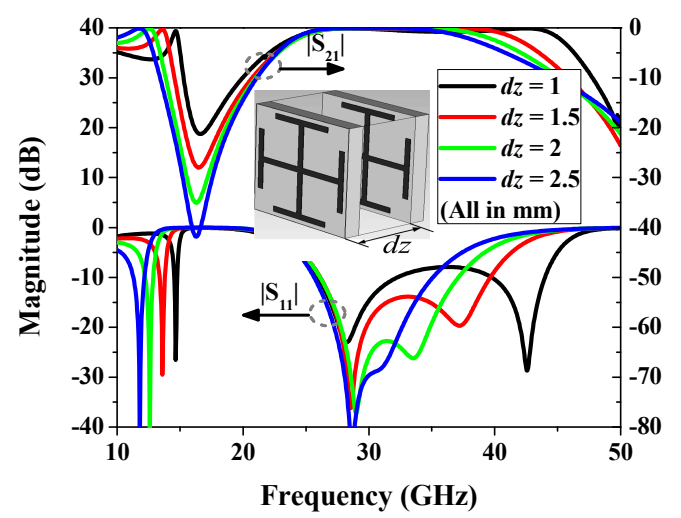

(a)

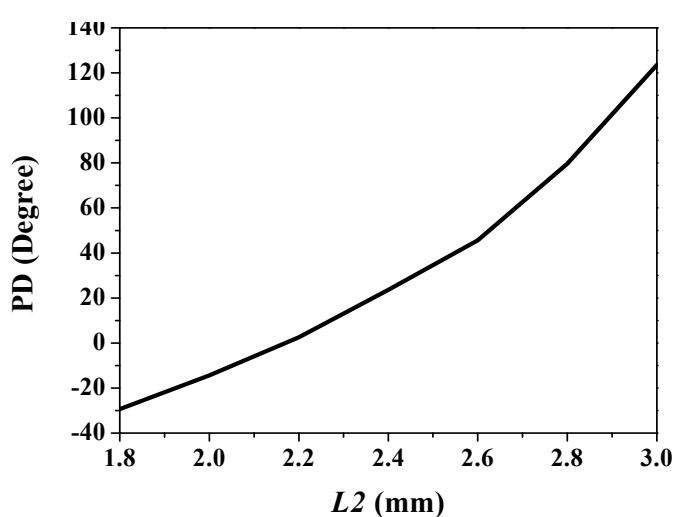

(b)

Fig.5. (a) Two-layer JC's transmission with variant spacing distance $d z$; (b) the $P D$ against $L 2(d z=2 \mathrm{~mm})$

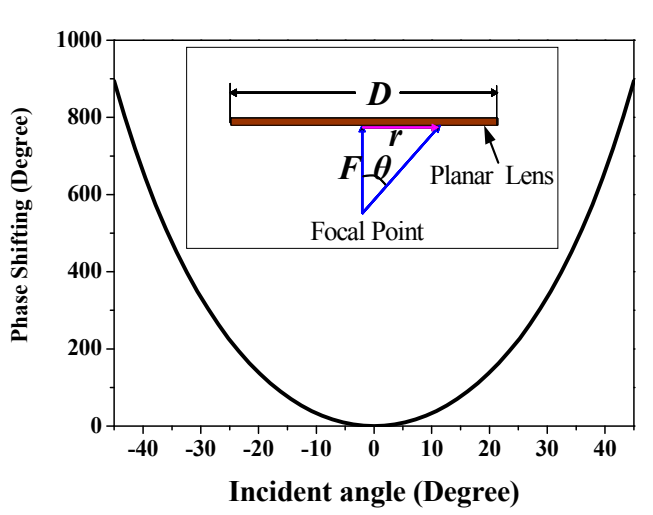

(a)

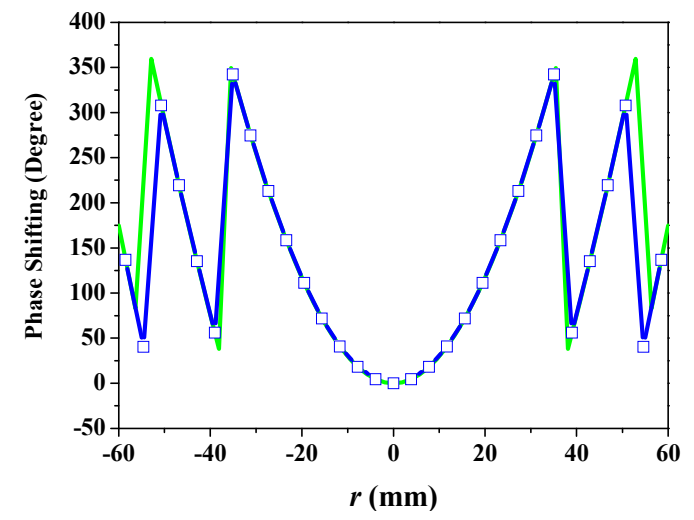

(b)

Fig.6. (a) The planar lens model and phase shifting caused by path difference, (b) Phase shifting for zone-plate lens 